\title{
Chimeric mouse models for lung adenocarcinomas
}

\section{Yi-Rong Chen}

Division of Molecular \& Genomic Medicine, National Health Research Institutes, 35 Keyan Road, Zhunan, Miaoli County 350, Taiwan = Tel.: +886 37246166 ext. 35311 = Fax: +886 37586459 m yrchen@nhri.org.tw

Evaluation of: Zhou Y, Rideout WM 3rd, Zi T et al:: Chimeric mouse tumor models reveal differences in pathway activation between ERBB family- and KRASdependent lung adenocarcinomas. Nat. Biotechnol. 28(1), 71-78 (2010). EGFR and KRAS mutations are among the most frequent oncogenic mutations in non-small-cell lung cancer. However, these two mutations are associated with distinct clinical presentations. The underlying mechanisms for these differences are unclear. This study by Zhou et al, examines lung cancer formation induced by oncogenic mutants of ERBB, EGFR and HER2, as well as KRAS, using a novel chimeric mouse approach. They demonstrate that the PI3K/Akt pathway is preferentially activated in $E R B B$-induced tumors while $K R A S$-induced lung cancer demonstrates strong activation of the c-Jun N-terminal kinase pathway. This result suggests that, despite the involvement of KRAS and ERBB receptors in the same signaling pathway, oncogenic KRAS and ERBB mutants utilize different cellular signals to induce cancer formation.

\section{Summary of methods \& results}

Zhou et al. initially generated transgenic mouse lines for lung-specific and inducible expression of wild-type or V659E mutants of human HER2 gene. In these mice, HER2 expression can be induced in bronchiolar epithelial cells by doxycycline. However, those transgenic mice developed severe epithelial hyperplasia and progressive respiratory distress in the 2 weeks after receiving doxycycline and required euthanasia. This prevented further analyses of HER2-induced oncogenesis. To overcome the limitation of conventional transgenic models, the authors used engineered embryonic stem (ES) cells and a chimera approach to produce mice that were mosaic for engineered oncogenes [1]. The ES cells underwent a series of genetic manipulations before the blastocyst injection. At each step, the ES lines were tested for the preservation of pluripotency and the functional efficacy of introduced genetic elements. First, two rounds of targeted recombination were used to inactivate the Ink4a/Arf tumor suppressor gene. The resultant ES cells were then cotransfected with CCSP-rtTA, a tetOluciferase transgene and a PGK-puromycin selection cassette. The puromycin-resistant ES cells, which expressed luciferase in response to doxycycline, were then transfected with $t e t O-H E R 2^{V 659 E}$ and $P G K$-hygromycin constructs. Those hygromycin-resistant ES cells that demonstrated robust doxycycline-driven and lung-specific $H E R 2^{V 659 E}$ expression (HER2 $2^{V 659 E}$ ES cells) were repetitively used to generate chimeric mice. $H E R 2^{V 659 E}$ ES cell chimeras developed lung tumors, which histologically resembled invasive adenocarcinomas, between 2 months and 1 year after starting doxycycline treatment at 4 weeks of age. Those tumors expressed human HER2 $2^{\mathrm{V} 659 \mathrm{E}}$, cytokeratin and surfactant protein $\mathrm{C}$, indicating that the tumors originated from lung epithelial cells descended from the genetically modified ES cells.

The same approach was used to generate $P I K 3 C A^{m y r}$ (myristylated $\left.\mathrm{p} 110 \alpha\right), E G F R^{W T}$, $E G F R^{L 858 R}$ and $K R A S^{G 12 V}$-driven chimera models. PIK3CA $A^{m y r}$ and $E G F R^{W T}$-driven models developed hyperplasia but no malignancy. By contrast, $E G F R^{L 858 R}$ and $K R A S^{G 12 V}$-driven models developed invasive adenocarcinoma within 4-12 months of doxycycline treatment, with mean tumor latency of 8 and 9 months, respectively. The tumors developed in EGFR and KRAS mutant chimera demonstrated histological features similar to those developed in $H E R 2^{V 659 E}$ mice. Treatment with an EGFR inhibitor, AV412, caused decreases in tumor burden in $E G F R^{L 858 R}$ mice but not in $K R A S^{G 12 V}$ mice, consistent with the clinical findings in human lung adenocarcinomas. Most interestingly, Akt phosphorylation of S473 and T308 residues was highly elevated in $H E R 2^{V G 59 E}$ and $E G F R^{L 858 R}$-dependent tumors but not in $K R A S^{G I 2 V}$-dependent tumors. On the other hand, JNK phosphorylation was increased in $K R A S^{G 12 V_{-}}$, but not in $H E R 2^{V 659 E}$ - and $E G F R^{L 858 R}$-dependent tumors.

\section{Discussion}

A combination of tissue-specific and tetracycline-inducible systems has been successfully used to generate EGFR mutant-triggered lung
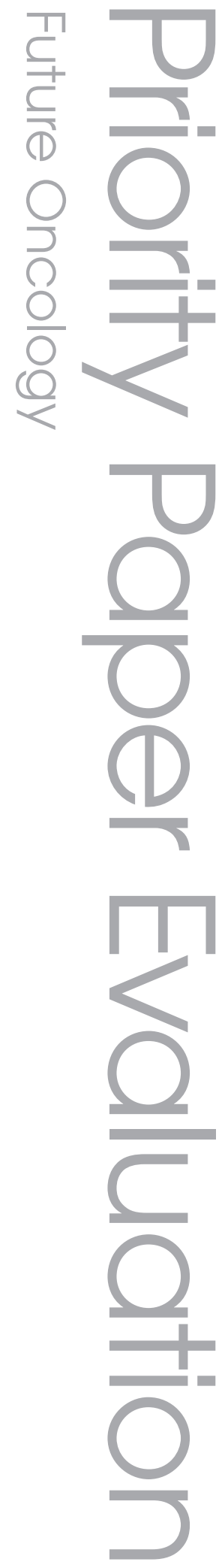

Keywords

- animal model $=E G F R$ - KRAS

= lung adenocarcinoma

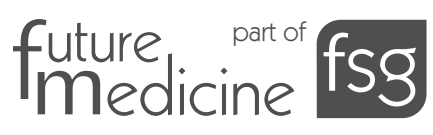


adenocarcinoma models. Tumors appear quickly after the administration of doxycycline in these animals [2,3]. Also, various transgenic models have been established to examine lung adenocarcinoma induced by KRAS mutation [4,5]. Zhou et al. demonstrated that, using a conventional transgenic approach, they failed to induce lung adenocarcinoma formation by expressing $H E R 2^{V 659 E}$ in mouse lung epithelial cells owing to acute adverse effects. This work did not explain the reason why HER2 $2^{V 659 E}$, in contrast to KRAS and EGFR mutants, demonstrated this specific characteristic. However, using engineered ES cells, the authors successfully overcame this problem and induced $H E R 2^{V 659 E}$-triggered lung cancer in chimeric mouse models. This study has provided a feasible approach that can be applied to other oncogenes that cause severe adverse effects when expressed universally in a particular tissue.

In contrast to previous animal studies, Zhou et al. revealed the distinct patterns of oncogenic signaling in $K R A S$ and $E R B B$ chimeric tumor models. The PI3K/Akt pathway is highly elevated in $H E R 2^{V 659 E}$ and $E G F R^{L 858 R}$-dependent tumors but not in $K R A S^{G 12 V}$-dependent tumors. By contrast, JNK phosphorylation was increased in $K R A S^{G 12 V}$-dependent tumors. This interesting phenomenon is most likely not unique to the reported chimera animal models. The convenience of ES cell-based chimeric mouse models may have facilitated the side-by-side comparison and revealed the differential regulation of cell signaling by different oncogenes.

One potential problem for this chimera mice approach is the wide range of disease severity and of the latency from starting doxycycline treatment to tumor onset (2-12 months in $H E R 2^{V 659 E}$ mice and 4-12 months in EGFR ${ }^{L 858 R}$ and $K R A S^{G 12 V}$ mice). Heterogeneity in disease progression may complicate the application of these models for testing preventive or therapeutic agents. This problem is most likely derived from the variations in chimera levels among the animals. If the severity of cancer formation can be predicted from a straightforward indicator of chimerism, for example, coat color, then the problem can be solved. However, the authors presented results demonstrating that the percentage of chimerism in coat color did not parallel the chimerism in the lung tissues. Therefore, this issue remains to be addressed.

\section{Future perspective}

EGFR mutations are frequently found in nonsmall-cell lung cancer (NSCLC) patients in east
Asian populations but not in Western countries, while the prevalence of the KRAS mutation is the opposite. Also, KRAS mutation can be found in various kinds of cancers. By contrast, $E G F R$ mutations are particularly frequent in lung adenocarcinoma, but rare in other types of cancers. Furthermore, these two mutations are mutually exclusive in NSCLC patients $[6,7]$. There is no reason to speculate that a certain mutation predominantly occurs in one tissue (or in one ethnic population), but not in the others. We can assume that a particular oncogenic mutation is suitable for certain cellular contexts, and its retention and continuous propagation will preferentially occur in these types of tumor cells. Because cellular contexts are distinct in different tissues (or in different ethnic populations), different oncogenic mutations will associate with distinct clinical features. The finding by Zhou et al. is consistent with this notion. Further analyses using chimera models may prove that different oncogenic mutations, through providing distinct patterns of signaling, induce various types of cancers in the animals.

Targeting therapy against molecules involved in tumor development and progression is a subject of extensive ongoing research. However, the prospect of developing targeted therapy is reduced owing to the emergence of drug resistance. For example, although EGFR-targeting therapy has been successful in treating NSCLC with EGFR mutations, recurrent resistant tumors often developed in EGFR inhibitor-treated patients. Currently, no appropriate animal models can be used to study the drug resistance problem. Similar to previous EGFR mutanttriggered lung adenocarcinoma models $[2,3]$, the chimeric mice reported by Zhou et al. developed invasive adenocarcinoma upon continuous doxycycline treatment. Those tumors lost oncogene expression and demonstrated decreased proliferation and increased apoptosis after withdrawal of doxycycline. Moreover, EGFR ${ }^{L 858 R}$ chimeric mice demonstrated tumor regression upon treatment with an EGFR inhibitor. These data indicate that the initiation and maintenance of the tumors requires the continuous presence of those oncogenes or their activity. However, it will be much more interesting to know if any of these animals can develop recurrent tumors after the start of inhibitor treatment or the withdrawal of doxycycline. This will indicate that tumors independent of the primary oncogenic signaling can be developed in these models. If that is the case, these animal models may be used to examine the mechanisms of resistance to targeting therapy. 


\section{Executive summary}

\section{Study design}

- Genetically engineered embryonic stem cells were used to generate chimera mice for doxycycline-inducible lung cancer studies.

- The end points were tumor incidence, histology, microarray analyses and immunohistochemistry examination of signaling pathways.

\section{Results}

- Adenocarcinomas formed in chimera mice containing HER2, KRAS or EGF receptor oncogenes exhibited features of advanced malignancies.

- Treatment of $E G F R^{L 858 R}$, but not the KRASG12V, chimeric models with an EGF receptor inhibitor resulted in near complete tumor regression.

- PI3K/Akt pathway activation is unique to ERBB family tumors whereas KRAS-driven tumors demonstrate activation of the JNK/SAPK pathway.

\section{Conclusion}

- Embryonic stem-cell-based chimera models can be used to examine oncogene-induced cancer formation when a conventional transgenic approach has acute adverse effects on animals. KRAS and ERBB mutants induce different cell signaling pathways in lung adenocarcinoma tissues, indicating different treatment options for cancers induced by different oncogenes.

\section{Financial \& competing} interests disclosure

The author has no relevant affliations or financial involvement with any organization or entity with a financial interest in or financial conflict with the subject matter or materials discussed in the manuscript. This includes employment, consultancies, honoraria, stock ownership or options, expert testimony, grants or patents received or pending, or royalties.

No writing assistance was utilized in the production of this manuscript.

\section{Bibliography}

Papers of special note have been highlighted as: - of interest

1. Zhou Y, Rideout WM 3rd, Zi T et al:: Chimeric mouse tumor models reveal differences in pathway activation between $E R B B$ family- and KRAS-dependent lung adenocarcinomas. Nat. Biotechnol. 28, 71-78 (2009).

2. Ji H, Li D, Chen L et al.: The impact of human EGFR kinase domain mutations on lung tumorigenesis and in vivo sensitivity to EGFR-targeted therapies. Cancer Cell 9, 485-495 (2006).
- Along with [3], uses transgenic animal models to demonstrate that inducible expression of EGFR mutants in mouse lung epithelial cells cause rapid lung adenocarcinoma formation. Also demonstrates that withdrawal of the inducing agent, doxcycyline, or administration of EGFR inhibitors results in rapid tumor regression. These animal models are useful in developing therapeutic agents against lung cancer harboring EGFR mutations.

3. Politi K, Zakowski MF, Fan P-D, Schondeld EA, Pao W, Varmus HE: Lung adenocarcinomas induced in mice by mutant EGFR receptors found in human lung cancers respond to a tyrosine kinase inhibitor or to down-regulation of the receptors. Genes Dev. 20, 1496-1510 (2006).

- Along with [2], uses transgenic animal models to demonstrate that inducible expression of EGFR mutants in mouse lung epithelial cells cause rapid lung adenocarcinoma formation. Also demonstrates that withdrawal of the inducing agent, doxcycyline, or administration of EGFR inhibitors results in rapid tumor regression. These animal models are useful in developing therapeutic agents against lung cancer harboring EGFR mutations.

4. Johnson L, Mercer K, Greenbaum D et al: : Somatic activation of the $K$-Ras oncogene causes early onset lung cancer in mice. Nature 410, 1111-1116 (2001).

5. Guerra C, Mijimolle N, Dhawahir A et al:: Tumor induction by an endogenous $K$-Ras oncogene is highly dependent on cellular context. Cancer Cell 4, 111-120 (2003).

6. Toschi L, Cappuzzo F: Understanding the new genetics of responsiveness to epidermal growth factor receptor tyrosine kinase inhibitors. Oncologist 12, 211-220 (2007).

7. Shigematsu H, Gazdar AF: Somatic mutations of epidermal growth factor receptor signaling pathway in lung cancers. Int. J. Cancer 118, 257-262 (2006). 